\title{
Searches for charginos and neutralinos in ATLAS
}

\section{Luigi Longo*, on behalf of the ATLAS Collaboration}

CERN, Esplanade des Particules 1, 1211 Meyrin, Switzerland

E-mail: luigi.longo@cern.ch

Supersymmetry (SUSY) is one of the most interesting theories describing physics beyond the Standard Model. An extensive program of SUSY searches has been consequently performed by the ATLAS experiment with the LHC data collected during Run-2. In this work, the latest ATLAS searches for charginos and neutralinos are reported. The data are found to be consistent with the Standard Model and limits at 95\% confidence level on the masses of the hypothesized particles are set for the different considered models.

Puebla, Mexico

\footnotetext{
${ }^{*}$ Speaker.
} 


\section{Introduction}

Despite the large number of experimental proofs showing the predictive power of the Standard Model (SM) [1-6], there are still several arguments suggesting SM is an effective theory valid up to a given energy scale. The hierarchy problem, the matter-antimatter asymmetry, the nature of the dark matter (DM) or the muon's anomalous magnetic moment are some of the open questions that Standard Model does not address. Between the possible SM extensions, Supersymmetry (SUSY) [7-12] is one of the most interesting theories since it provides a natural solution to the hierarchy problem and a viable DM candidate, for example. In its minimal formulation, the Minimal Supersymmetric Standard Model (MSSM) [13], it predicts a new bosonic (fermionic) partner for each fermionic (bosonic) SM particle with identical quantum number, except for a difference of half unit of spin $(S)$. An additional Higgs doublet is also introduced together with its supersymmetric partner and a new multiplicative quantum number, $R$-parity, is defined as $R=(-1)^{3(B-L)+2 S}$ with $B$ as baryon number and $L$ as lepton number. The possibility of a supersymmetric dark matter [14,15] candidate is closely related to the $R$-parity conservation [16]; in fact, under this assumption, the lightest supersymmetric particle (LSP) is stable and represents a possible DM candidate.

In this proceeding the latest SUSY analyses performed by the ATLAS [17] collaboration, considering the Large Hadron Collider (LHC) [18] Run-2 data $\left(139 \mathrm{fb}^{-1}\right)$, for the search of the mass eigenstates ${ }^{1}$ of the superpartners of the Higgs and electroweak gauge bosons, neutralinos $\left(\tilde{\chi}_{i=1,2,3,4}^{0}\right)$ and charginos $\left(\tilde{\chi}_{i=1,2}^{ \pm}\right)$, in a $R$-parity conserving scenario are presented.

\subsection{Signal models}

The investigated SUSY scenarios assume the lightest neutralino as LSP and the direct production of $\tilde{\chi}_{1}^{ \pm} \tilde{\chi}_{1}^{ \pm}$or $\tilde{\chi}_{1}^{ \pm} \tilde{\chi}_{2}^{0}$, considering for the latter the produced particles to be degenerate in mass. In all the models $\tilde{\chi}_{1}^{ \pm}$decays into a $W$ boson and $\tilde{\chi}_{1}^{0}$, while 2 different decay modes are considered for $\tilde{\chi}_{2}^{0}$ : one involving the Higgs boson $\left(\tilde{\chi}_{2}^{0} \rightarrow h \tilde{\chi}_{1}^{0}\right)$ and the other the $Z$ boson $\left(\tilde{\chi}_{2}^{0} \rightarrow Z \tilde{\chi}_{1}^{0}\right)$; nevertheless only one of the 2 decays were allowed in each signal model.

Three additional models were also studied, the so-called compressed scenarios, where the mass differences between the involved particles are lower than $Z$ and $W$ masses:

- Higgsino-like case where a direct production of $\tilde{\chi}_{1}^{ \pm} \tilde{\chi}_{2}^{0}$ is assumed; $\tilde{\chi}_{1}^{ \pm}, \tilde{\chi}_{2}^{0}, \tilde{\chi}_{1}^{0}$ are a triplet of Higgsino-like states with $m\left(\tilde{\chi}_{1}^{ \pm}\right)=\frac{1}{2}\left[m\left(\tilde{\chi}_{2}^{0}\right)+m\left(\tilde{\chi}_{1}^{0}\right)\right]$.

- Wino/Bino-like case where there is always a direct production of $\tilde{\chi}_{1}^{ \pm} \tilde{\chi}_{2}^{0}$ but $\tilde{\chi}_{1}^{ \pm} \tilde{\chi}_{2}^{0}$ are degenerate pure wino states with $\tilde{\chi}_{1}^{0}$ being pure bino ${ }^{2}$.

- Sleptons case where there is a direct production of selectrons and smuons, which are degenerate in mass, and $\tilde{\chi}_{1}^{0}$ is a pure bino state.

In the first 2 models $\tilde{\chi}_{2}^{0}$ is allowed to decay only into $Z \tilde{\chi}_{1}^{0}$; for the third one, only the slepton decay into $\tilde{\chi}_{1}^{0}$ and the corresponding SM partner is considered.

\footnotetext{
${ }^{1}$ All the mass eigenstates are ordered by increasing mass.

${ }^{2}$ For the Wino/Bino-like scenario the process kinematic depends on the relative sign between $\tilde{\chi}_{2}^{0}$ and $\tilde{\chi}_{1}^{0}$.
} 


\section{2. $\tilde{\chi}_{1}^{ \pm} \tilde{\chi}_{2}^{0}$ direct production}

Depending on the assumed decay of $\tilde{\chi}_{2}^{0}$ and on the decay modes of $W, Z$ and $h$, different analysis strategies were considered for the signal models targeting a direct production of $\tilde{\chi}_{1}^{ \pm} \tilde{\chi}_{2}^{0}[19-$ 21].

In the case of $\tilde{\chi}_{2}^{0}$ decaying into a Higgs boson and $\tilde{\chi}_{1}^{0}$, final states with $2 b$-quarks, 2 photons $(\gamma)$ or multi-leptons coming from $h$ are expected and they were considered separately [19]. A different trigger choice was also used, respectively missing transverse energy $\left(E_{\mathrm{T}}^{\mathrm{miss}}\right)$, diphoton and leptonic triggers. For all the analysis strategies the basic idea was to identify events with a Higgs candidate, selecting an invariant mass of $2 b$-quarks $\left(m_{b b}\right)$ or of 2 photons $\left(m_{\gamma \gamma}\right)$ close to the $h$ mass, or applying an upper cut on the invariant mass between the jet (or the 2 leading jets) and the closest lepton $\left(m_{l j}\right)$ which is a good estimator of the Higgs boson mass (Figure 1). The analyses
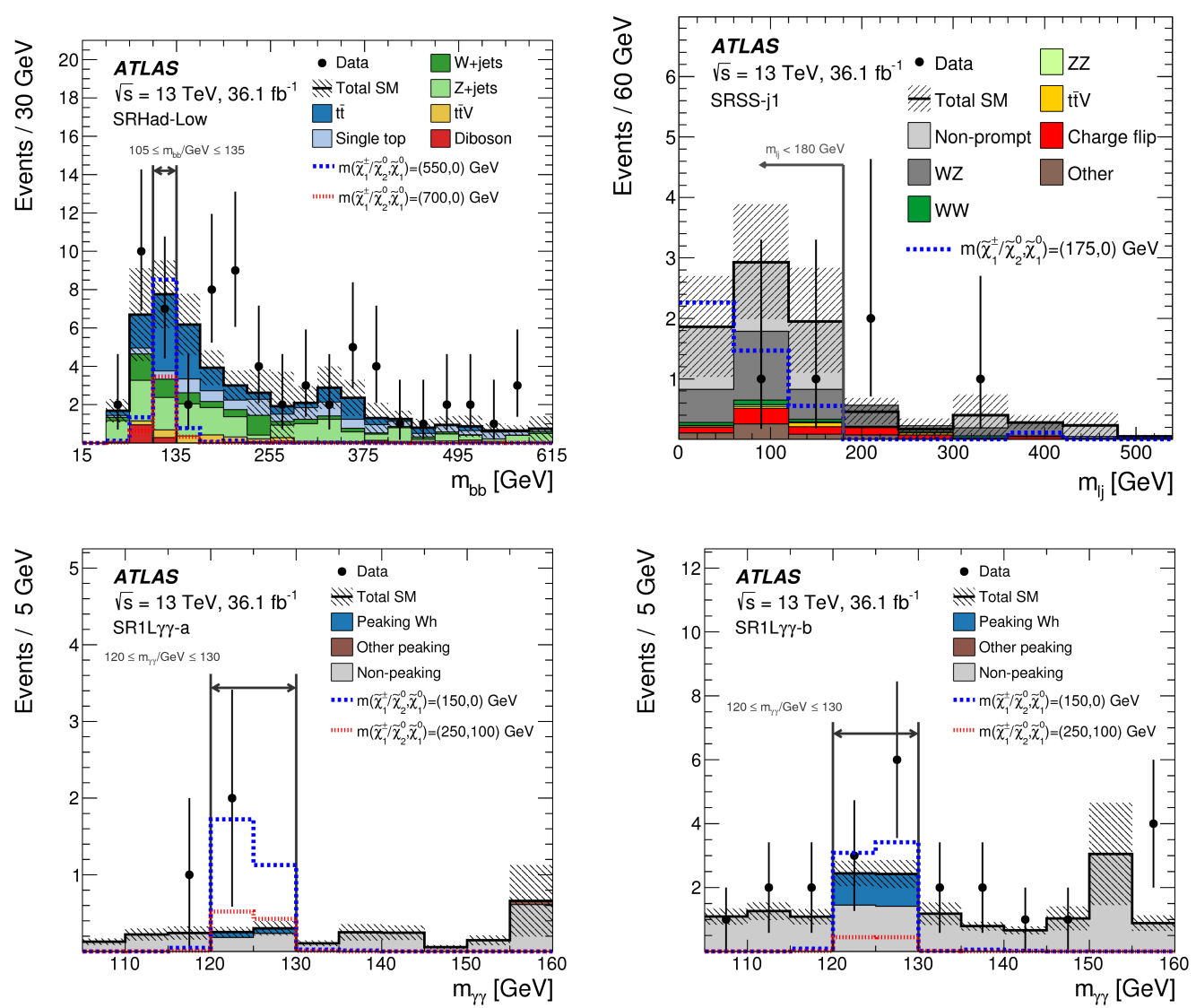

Figure 1: Distributions of $m_{b b}$ (top left), $m_{l j}$ (top right) and $m_{\gamma \gamma}$ for some of the signal regions defined in Ref. [19], obtained with an integrated luminosity of $36.1 \mathrm{fb}^{-1}$. A mild excess is present for the signal regions targeting a diphotons decay of the Higgs boson (bottom plots).

were done with $36.1 \mathrm{fb}^{-1}$ data collected during 2015 and 2016. A mild excess was observed for the diphoton case and a follow up was done with the full Run-2 data, following exactly the same selection of the $36.1 \mathrm{fb}^{-1}$ analysis, but no significant excess was found as shown in Figure 2 [20]. To be more sensitive to the signal models with $h \rightarrow \gamma \gamma$, the full Run-2 analysis performed also a 
series of unbinned likelihoot fits in different categories, which were based on different requests on the number of jets and leptons, the ratio between $E_{\mathrm{T}}^{\text {miss }}$ and scalar sum of the transverse momenta of calibrated objects, the dijets invariant mass. No significant excess was found in all the analyses
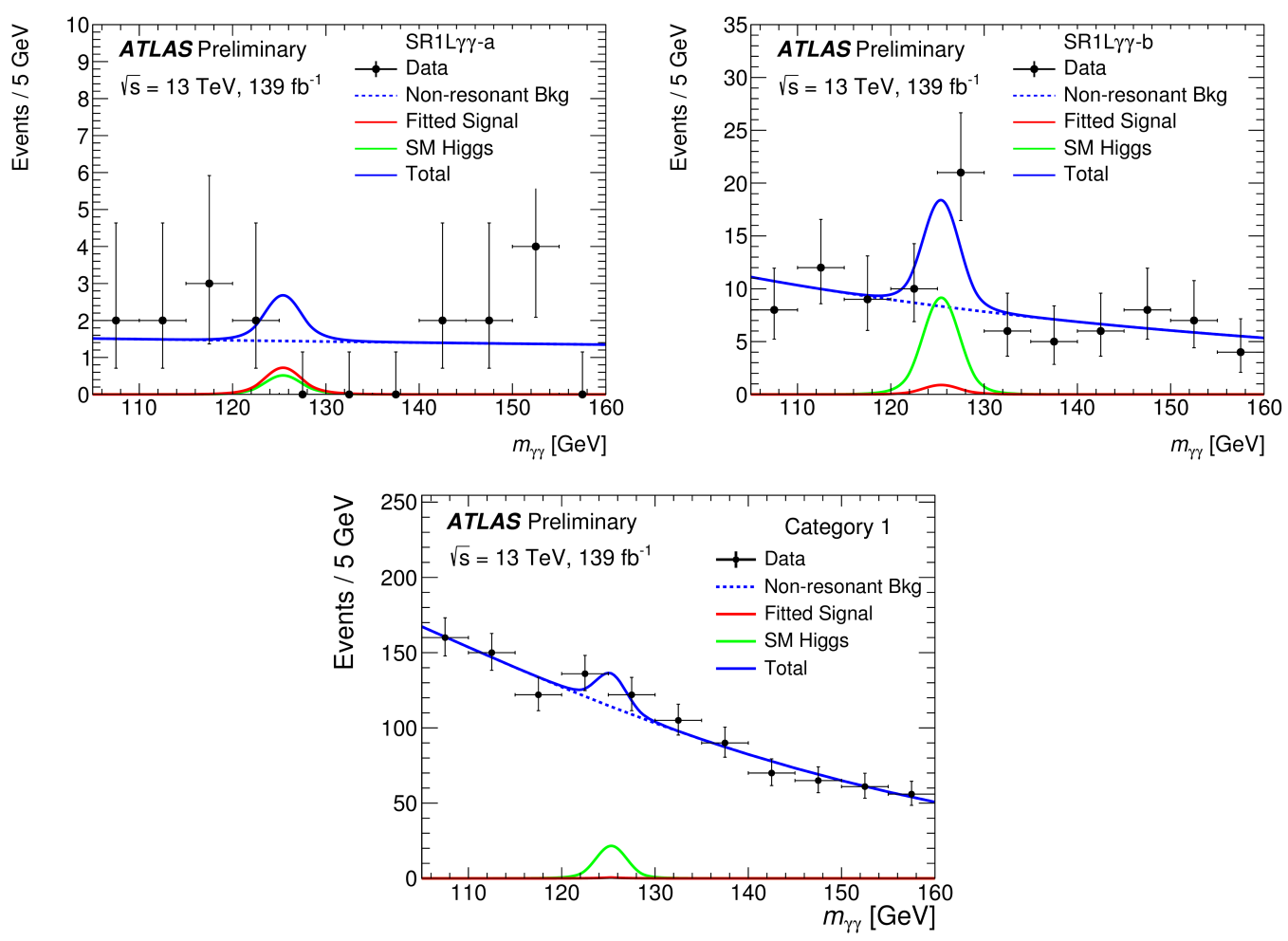

Figure 2: Unbinned likelihood fit for the signal regions targeting the mild excess observed in Ref. [19] (top plots) and for one of the categories considered in Ref. [20] (bottom plot). No significant excess is present once the full Run-2 data are considered.

and exclusion limits at $95 \%$ confidence level (CL) were set in the mass plane $m\left(\tilde{\chi}_{1}^{ \pm} / \tilde{\chi}_{2}^{0}\right)-m\left(\tilde{\chi}_{1}^{0}\right)$ (Figure 3).

For the case where $\tilde{\chi}_{2}^{0}$ decays into a $Z$ boson and $\tilde{\chi}_{1}^{0}$, an analysis targeting the full Run-2 data was developed [21]. It looked at three-lepton final states and was a follow-up of the Recursive Jigsaw Reconstruction (RJR) based analysis, which shows an excess with 2015 and 2016 data [23]. RJR variables were translated in conventional variables and a selection nearly identical to the $36.1 \mathrm{fb}^{-1}$ analyses was used. However no significant excess was found and limits at 95\% CL were set (Figure 4). 


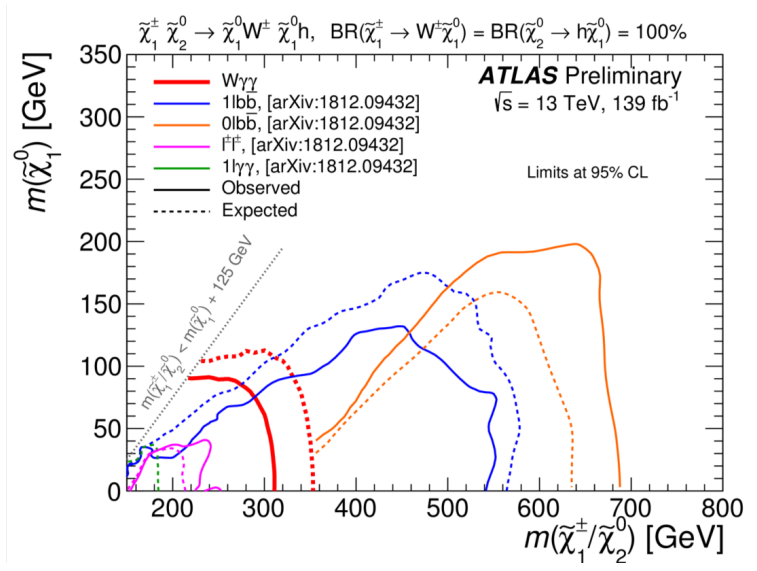

Figure 3: The $95 \% \mathrm{CL}$ exclusion limits on $\tilde{\chi}_{1}^{ \pm} \tilde{\chi}_{2}^{0}$ with $\tilde{\chi}_{1}^{ \pm} \rightarrow W^{ \pm} \tilde{\chi}_{1}^{0}$ and $\tilde{\chi}_{2}^{0} \rightarrow h \tilde{\chi}_{1}^{0}$, as a function of $\tilde{\chi}_{1}^{ \pm} / \tilde{\chi}_{2}^{0}$ and $\tilde{\chi}_{1}^{0}$ masses, for the analysis strategies targeting in the final state $2 b$-quarks (1lbb and $\left.0 l b \bar{b}\right), 2$ photons ( $1 l \gamma \gamma$ and $W \gamma \gamma$ respectively for the analyses considering $36.1 \mathrm{fb}^{-1}$ and the full Run-2 dataset) and multileptons $\left(l^{ \pm} l^{ \pm}\right)[22]$.
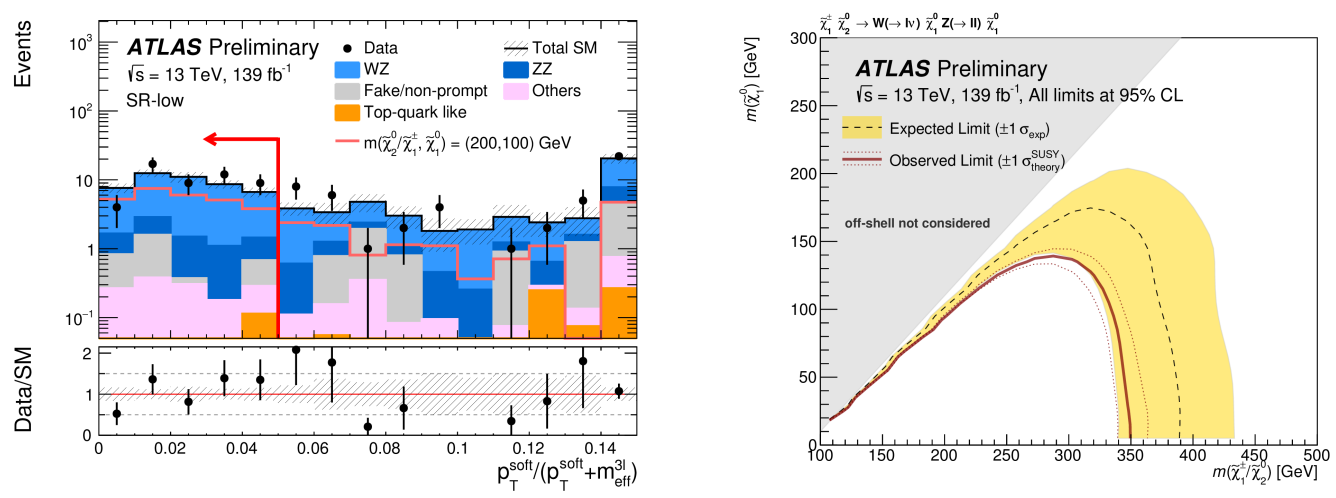

Figure 4: On the left, distribution of one of the variables defined to emulate RJR variables for one of the signal regions defined in Ref. [21]; $p_{\mathrm{T}}^{\text {soft }}$ and $m_{\mathrm{eff}}^{3 l}$ are respectively vectorial and scalar sum of transverse momenta of the 3 leptons and $E_{\mathrm{T}}^{\text {miss }}$ [21]. On the right, the $95 \% \mathrm{CL}$ exclusion limits on $\tilde{\chi}_{1}^{ \pm} \tilde{\chi}_{2}^{0}$ with $\tilde{\chi}_{1}^{ \pm} \rightarrow$ $W^{ \pm} \tilde{\chi}_{1}^{0}$ and $\tilde{\chi}_{2}^{0} \rightarrow Z \tilde{\chi}_{1}^{0}$, as a function of $\tilde{\chi}_{1}^{ \pm} / \tilde{\chi}_{2}^{0}$ and $\tilde{\chi}_{1}^{0}$ masses for the analysis in Ref. [21].

\section{3. $\tilde{\chi}_{1}^{ \pm} \tilde{\chi}_{1}^{ \pm}$direct production}

The search for a direct pair production of the lightest chargino was developed considering a dileptonic final state and vetoing $b$-tagged jets with full Run-2 data [24]. The analysis was based on the stransverse mass $[25,26]$ defined as

$$
m_{\mathrm{T} 2}\left(\mathbf{p}_{\mathrm{T}, 1}, \mathbf{p}_{\mathrm{T}, 2}, \mathbf{q}_{\mathrm{T}}\right)=\min _{\mathbf{q}_{\mathrm{T}, 1}+\mathbf{q}_{\mathrm{T}, 2}=\mathbf{q}_{\mathrm{T}}}\left\{\max \left[m_{\mathrm{T}}\left(\mathbf{p}_{\mathrm{T}, 1}, \mathbf{q}_{\mathrm{T}, 1}\right), m_{\mathrm{T}}\left(\mathbf{p}_{\mathrm{T}, 2}, \mathbf{q}_{\mathrm{T}, 2}\right)\right]\right\}
$$

where $m_{\mathrm{T}}$ indicates the transverse mass, ${ }^{3} \mathbf{p}_{\mathrm{T}, 1}$ and $\mathbf{p}_{\mathrm{T}, 2}$ are the transverse momentum vectors of two particles (assumed to be massless), and $\mathbf{q}_{\mathrm{T}, 1}$ and $\mathbf{q}_{\mathrm{T}, 2}$ are vectors so that $\mathbf{q}_{\mathrm{T}}=\mathbf{q}_{\mathrm{T}, 1}+\mathbf{q}_{\mathrm{T}, 2}$, with $\mathbf{q}_{\mathrm{T}}$

\footnotetext{
${ }^{3}$ The transverse mass is defined by the equation $m_{\mathrm{T}}=\sqrt{2\left|\mathbf{p}_{\mathrm{T}, 1}\right|\left|\mathbf{p}_{\mathrm{T}, 2}\right|(1-\cos (\Delta \phi))}$, where $\Delta \phi$ is the angle between the particles with transverse momenta $\mathbf{p}_{\mathrm{T}, 1}$ and $\mathbf{p}_{\mathrm{T}, 2}$ in the plane perpendicular to the beam axis.
} 
assumed to be the missing transverse momentum of the system. Different categories were defined, based on the lepton flavour and jet multiplicity (maximum 1 jet was allowed) and a binning on $m_{\mathrm{T} 2}$ was performed to fit the different signal models (Figure 5). No significant excess was found and limits at $95 \% \mathrm{CL}$ on the mass plane $m\left(\tilde{\chi}_{1}^{ \pm}\right)-m\left(\tilde{\chi}_{1}^{0}\right)$ were set (Figure 5).
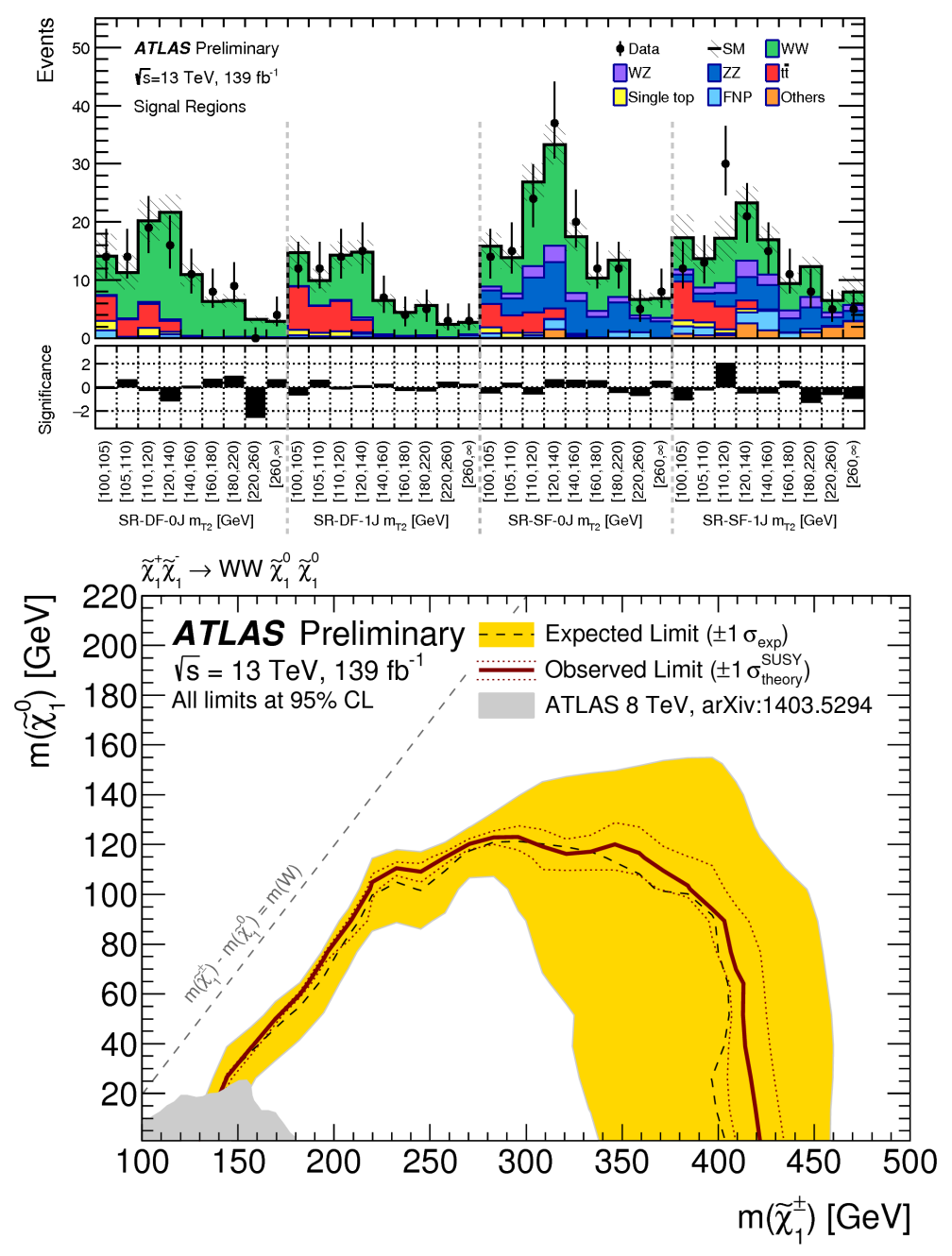

Figure 5: On the top, data/Monte Carlo event comparison for each of the bin considered for the given signal regions; the signal regions were defined considering the lepton flavour (same flavour, SF, or different flavour, $\mathrm{DF})$ and if no jet $(0 \mathrm{~J})$ or 1 jet $(1 \mathrm{~J})$ is present in the final state. On the bottom, the $95 \% \mathrm{CL}$ exclusion limits on the direct chargino pair production as a function of $\tilde{\chi}_{1}^{ \pm}$and $\tilde{\chi}_{1}^{0}$ masses [24].

Given the similar final state, the analysis was also interpreted for signal models where $\tilde{\chi}_{1}^{ \pm}$ decays via slepton (selectron, smuon or sneutrino) or a direct slepton (selectron or smuon) pair production is assumed. Figure 6 shows the limits obtained for the last 2 models. 

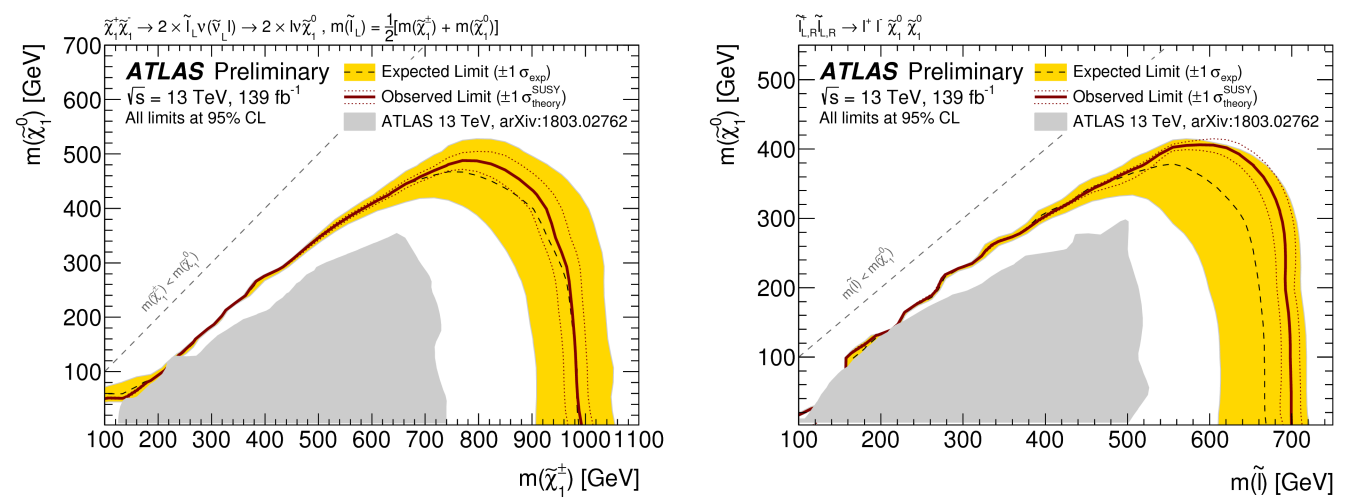

Figure 6: On the left, the $95 \% \mathrm{CL}$ exclusion limits on $\tilde{\chi}_{1}^{ \pm} \tilde{\chi}_{1}^{ \pm}$as a function of $\tilde{\chi}_{1}^{ \pm}$and $\tilde{\chi}_{1}^{0}$ masses, once it is assumed a $\tilde{\chi}_{1}^{ \pm}$decay into $\tilde{\chi}_{1}^{0}$ through the production of a slepton with a mass equal to the average mass between chargino and neutralino; a branching ratio of $50 \%$ is assumed for $\tilde{\chi}_{1}^{ \pm} \rightarrow \tilde{l} v$ or $\tilde{\chi}_{1}^{ \pm} \rightarrow \tilde{v} l$. On the right, the $95 \%$ CL exclusion limits on $\tilde{l} \tilde{l}$, as a function of $\tilde{l}$ and $\tilde{\chi}_{1}^{0}$ masses [24].

\section{Compressed scenarios}

In all the compressed scenarios [27], the final state is characterized by soft leptons and a $E_{\mathrm{T}}^{\text {miss }}$ trigger was used for the event selection.

For the Higgsino-like and Wino/Bino-like cases, the final states considered were with 2 leptons (2L) or 1 lepton plus 1 track (1L1T), with the candidate track having a transverse momentum of less than $5 \mathrm{GeV}$. For $2 \mathrm{~L}$ final state, 3 different categories based on $E_{\mathrm{T}}^{\text {miss }}$ and the ratio between the scalar sum of the leptons and $E_{\mathrm{T}}^{\text {miss }}$ were defined. Given the change in the dilepton (lepton-track) invariant mass $\left(m_{l l(\text { track })}\right)$ distribution on the basis of the signal model under study, a binning on $m_{l l(\text { track })}$ was done to fit the signals shape (Figure 7). Limits at $95 \% \mathrm{CL}$ were set on the sparticle masses given the absence of any significant excess (Figure 8).

For the sleptons scenario, instead, only a 2-lepton final state was considered and 2 signal regions were defined on $E_{\mathrm{T}}^{\text {miss }}$ basis. Due to the different shape observed in the signal $m_{\mathrm{T} 2}$ distribution, a binning on the stransverse mass was defined (Figure 9). Also in this case no significant excess was found and limits at $95 \% \mathrm{CL}$ were placed in the plane $\Delta m\left(\tilde{l}, \tilde{\chi}_{1}^{0}\right)-m\left(\tilde{l}_{L, R}\right)$ (Figure 10). 

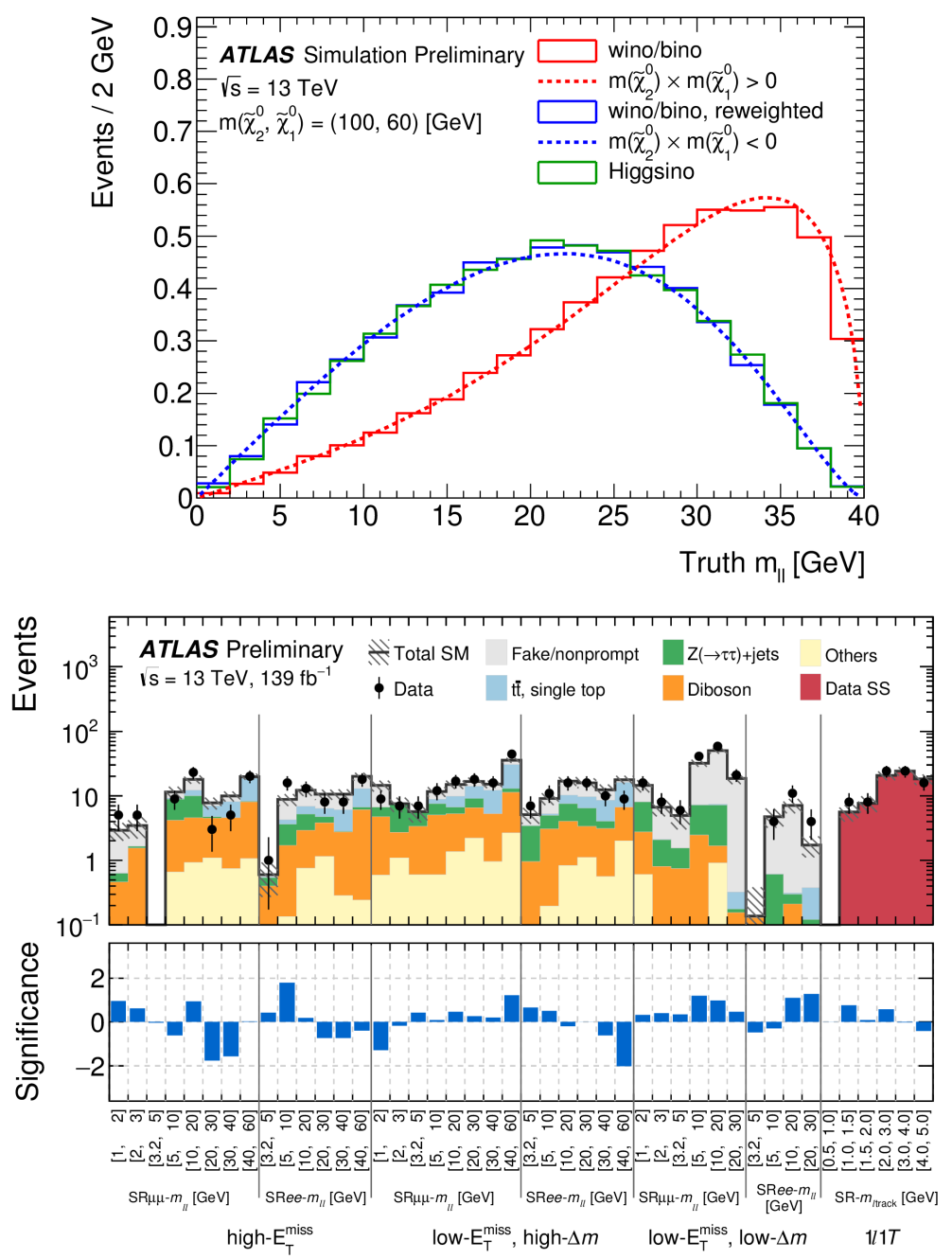

Figure 7: On the top, dilepton invariant mass for Higgsino and Wino/Bino simplified models. The endpoint of the distribution is determined by the difference between the masses of the $\tilde{\chi}_{2}^{0}$ and $\tilde{\chi}_{1}^{0}$; the product of signed mass eigenvalues $\left(m\left(\tilde{\chi}_{2}^{0}\right) \times m\left(\tilde{\chi}_{1}^{0}\right)\right)$ is negative for the Higgsino-like case and can be either negative or positive for the Wino/Bino-like case. On the bottom, data/Monte Carlo event comparison for each of the bin considered for the given signal regions; the signal regions were defined on the basis of the final state (2L or $1 \mathrm{~L} 1 \mathrm{~T}), E_{\mathrm{T}}^{\mathrm{miss}}$ and the flavour of the leptons (electrons or muons) [27]. 

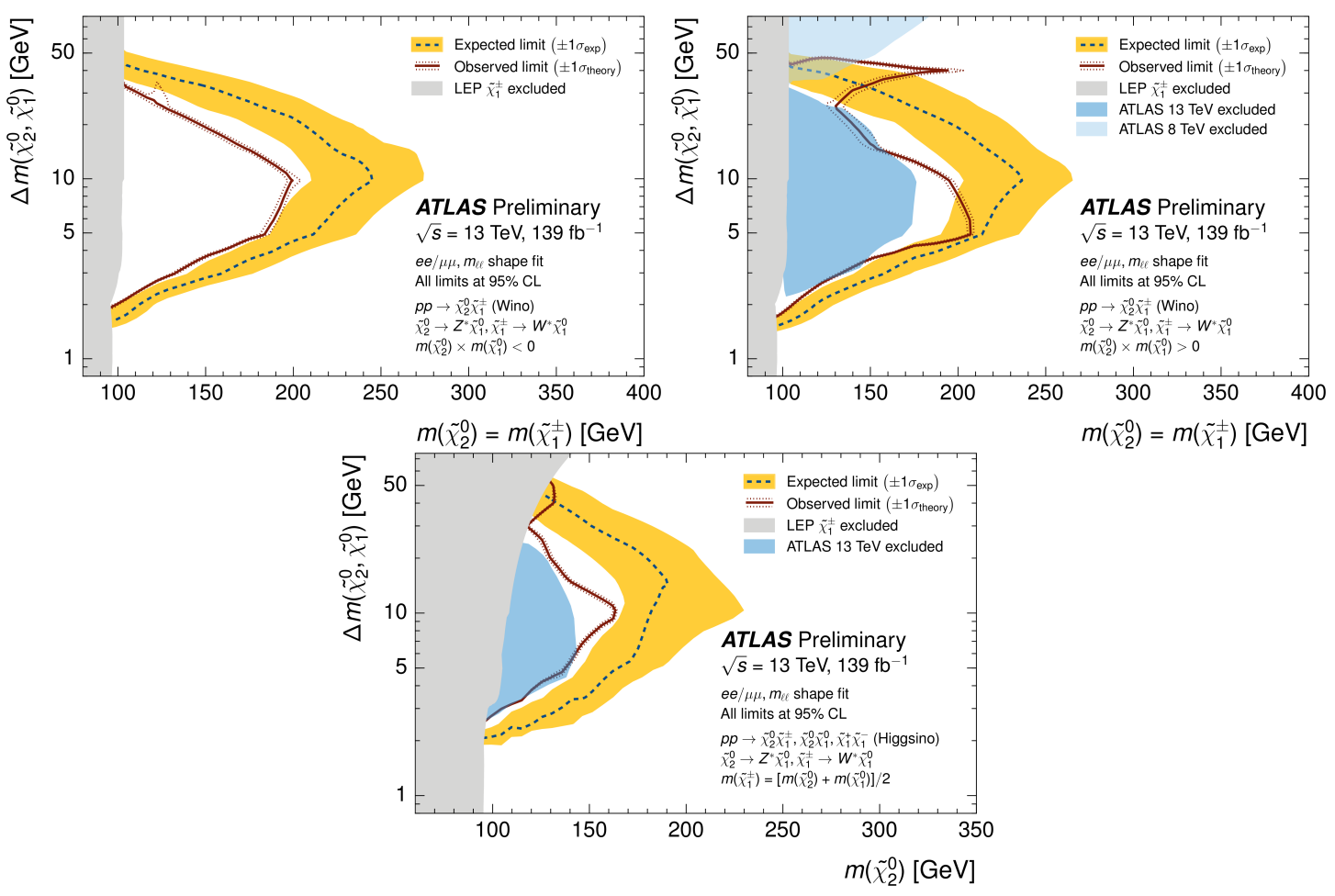

Figure 8: On the top, the 95\% CL exclusion limits for the Wino/Bino-like scenario as a function of $\tilde{\chi}_{1}^{ \pm} / \tilde{\chi}_{2}^{0}$ and $\tilde{\chi}_{1}^{0}$ masses, assuming a negative (left) or positive (right) sign between the 2 neutralino masses. On the bottom, the $95 \%$ CL exclusion limits for the Higgsino-like scenario as a function of $\tilde{\chi}_{2}^{0}$ mass and the splitting in mass between the 2 neutralinos [27]. The gray regions denote the lower chargino mass limit from LEP [28]. The blue regions indicates the limits from ATLAS Run-1 [29,30] and Run-2 [31].

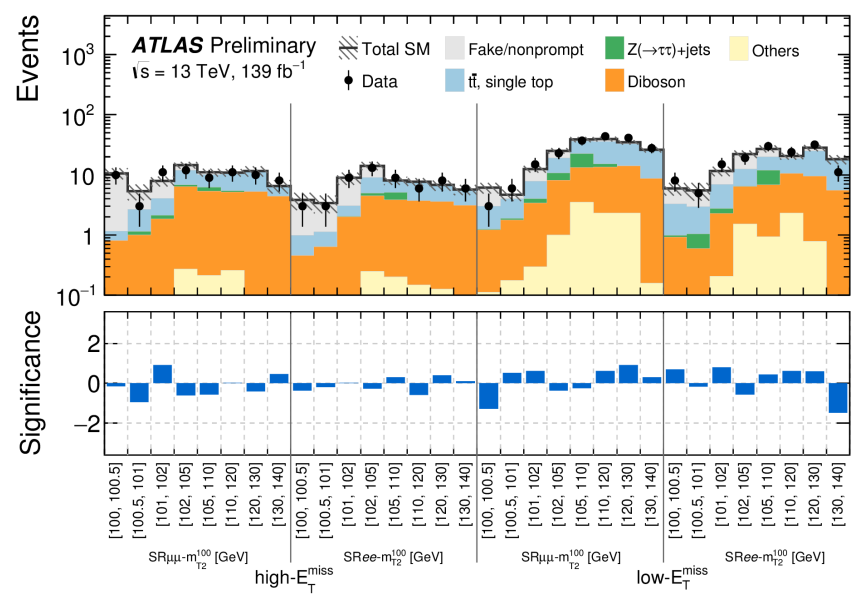

Figure 9: Data/Monte Carlo event comparison for each of the bin considered for the given signal regions; the signal regions were defined on the basis of $E_{\mathrm{T}}^{\text {miss }}$ and the flavour of the leptons (electrons or muons) [27]. 

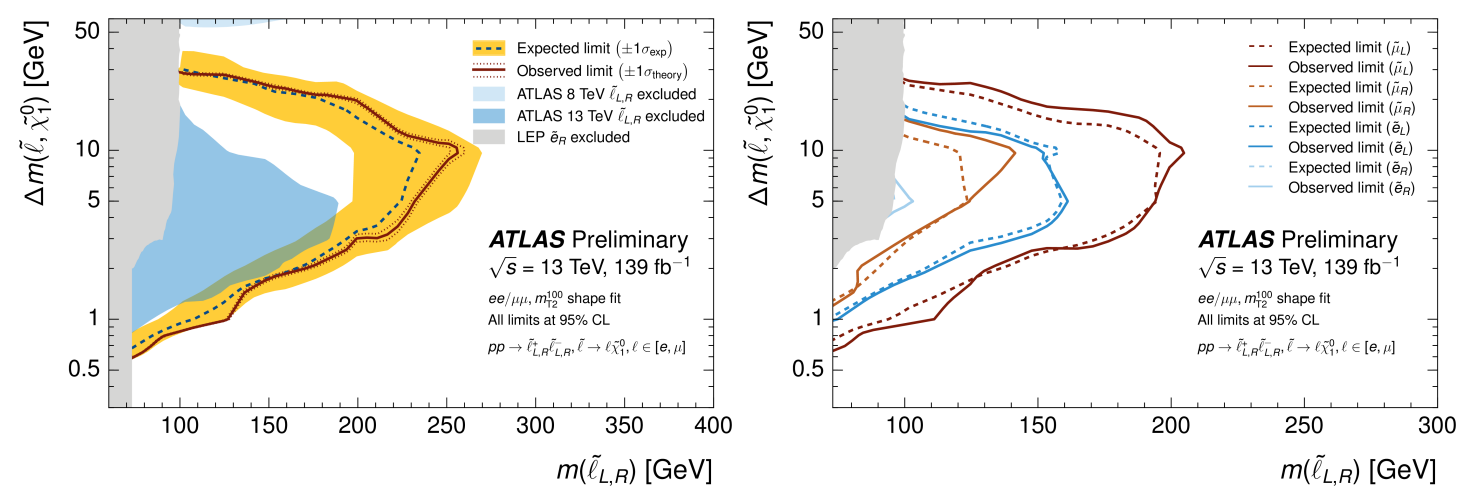

Figure 10: On the left, the 95\% CL exclusion limits for the sleptons scenario as a function of slepton mass and the splitting in mass between slepton and neutralino; right and left-handed selectron and smuon are considered to be degenerate in mass. On the right the 95\% CL exclusion limits for the slepton scenario once no degeneracy is assumed on the masses of the sleptons and the limits are presented separately for $\tilde{\mu}_{L}$, $\tilde{\mu}_{R}, \tilde{e}_{L}$ and $\tilde{e}_{R}$ [27]. The gray region is the $\tilde{e}_{R}$ limit from LEP [32]. The blue regions are the fourfold mass degenerate slepton limits from ATLAS Run-1 [29] and Run-2 [31].

\section{Conclusions}

ATLAS performed a comprehensive search for the electroweak pair production of chargino and neutralino with Run-2 data. The compressed scenarios were also addressed. No significant excess was found and limits on the SUSY particle masses were set for the specific models under study.

\section{References}

[1] S. L. Glashow, J. Iliopoulos, and L. Maiani; Weak Interactions with Lepton-Hadron Symmetry, Phys. Rev. D 2, 1285 (1970).

[2] S. Weinberg; A Model of Leptons, Phys. Rev. Lett. 19, 1264 (1967).

[3] A. Salam and J. C. Ward; Weak and Electromagnetic Interactions, Nuovo Cim (1959) 11:568.

[4] S. Glashow; Partial Symmetries of Weak Interactions, Nucl. Phys. 22 (1961).

[5] G. Altarelli; The physics of deep inelastic phenomena, Riv. Nuovo Cim. 4 (1974) 335.

[6] H. D. Politzer; Asymptotic freedom: An approach to strong interactions, Phys. Reports 14 (1974) 129.

[7] Y. Golfand and E. Likhtman; Extension of the algebra of poincare group generators and violation of $p$ invariance, JETP Lett. 13 (1971) 323-326.

[8] D. Volkov and V. Akulov; Is the neutrino a goldstone particle?, Phys. Lett. B 46, 109 (1973).

[9] J. Wess and B. Zumino; Supergauge transformations in four dimensions, Nucl. Phys. B 70, 39 (1974).

[10] J. Wess and B. Zumino; Supergauge invariant extension of quantum electrodynamics, Nucl. Phys. B 78, 1 (1974).

[11] S. Ferrara and B. Zumino; Supergauge invariant Yang-Mills theories, Nucl. Phys. B 79, 413 (1974).

[12] A. Salam and J. A. Strathdee; Super-symmetry and non-Abelian gauges, Phys. Lett. B 51, 353 (1974). 
[13] S. P. Martin; A Supersymmetry Primer, arXiv:hep-ph/9709356.

[14] H. Goldberg; Constraint on the Photino Mass from Cosmology, Phys. Rev. Lett. 50, 1419 (1983); Erratum 103, 099905 (2009).

[15] J. R. Ellis, J. S. Hagelin, D. V. Nanopoulos, K. A. Olive, and M. Srednicki; Supersymmetric relics from the big bang, Nucl. Phys. B238, 453 (1984).

[16] G. R. Farrar and P. Fayet; Phenomenology of the production, decay, and detection of new hadronic states associated with supersymmetry, Phys. Lett. B 76, 575 (1978).

[17] ATLAS Collaboration, 2008 JINST 3 S08003.

[18] L. Evans and P. Bryant (editors) 2008 JINST 3 S08001.

[19] ATLAS Collaboration, Search for chargino and neutralino production in final states with a Higgs boson and missing transverse momentum at $\sqrt{s}=13$ TeV with the ATLAS detector, Phys. Rev. D 100, 012006 (2019) [hep-ex/1812.09432].

[20] ATLAS Collaboration, ATLAS-CONF-2019-019, http://cdsweb.cern.ch/record/2676596.

[21] ATLAS Collaboration, ATLAS-CONF-2019-020, http://cdsweb.cern.ch/record/2676597.

[22] ATLAS Collaboration, http://atlas.web.cern.ch/Atlas/GROUPS/PHYSICS/CombinedSummaryPlots/SUSY/.

[23] ATLAS Collaboration, Search for chargino-neutralino production using recursive jigsaw reconstruction in final states with two or three charged leptons in proton-proton collisions at $\sqrt{s}=13$ TeV with the ATLAS detector, Phys. Rev. D 98, 092012 (2018) [hep-ex/1806.02293].

[24] ATLAS Collaboration, ATLAS-CONF-2019-008, http://cdsweb.cern.ch/record/2668387.

[25] C. G. Lester and D. J. Summers, Measuring masses of semiinvisibly decaying particles pair produced at hadron colliders, Phys. Lett. B 463 (1999) 99 [hep-ph/ 9906349 ].

[26] A. Barr, C. Lester and P. Stephens, $m(T 2)$ : The Truth behind the glamour, J. Phys. G 29 (2003) 2343 [hep-ph/0304226].

[27] ATLAS Collaboration, ATLAS-CONF-2019-014, http://cdsweb.cern.ch/record/2675954.

[28] ALEPH, DELPHI, L3, OPAL Experiments, Combined LEP Chargino Results, up to 208 GeV for low DM, LEPSUSYWG/02-04.1, 2002, url: http://lepsusy.web.cern.ch/lepsusy/www/ inoslowdmsummer02/charginolowdm_pub.html.

[29] ATLAS Collaboration, Search for direct production of charginos, neutralinos and sleptons in final states with two leptons and missing transverse momentum in pp collisions at $\sqrt{\mathrm{s}}=8 \mathrm{TeV}$ with the ATLAS detector, JHEP 05 (2014) 071 [hep-ex/1403.5294].

[30] ATLAS Collaboration, Search for direct production of charginos and neutralinos in events with three leptons and missing transverse momentum in $\sqrt{s}=8 \mathrm{TeV}$ pp collisions with the ATLAS detector, JHEP 04 (2014) 169 [hep-ex/1402 . 7029].

[31] ATLAS Collaboration, Search for electroweak production of supersymmetric states in scenarios with compressed mass spectra at $\sqrt{s}=13$ TeV with the ATLAS detector, Phys. Rev. D 97 (2018) 052010, [hep-ex/1712.08119].

[32] ALEPH, DELPHI, L3, OPAL Experiments, Combined LEP Selectron/Smuon/Stau Results, 183-208 $\mathrm{GeV}$, LEPSUSYWG/04-01.1, 2004, url: http://lepsusy.web.cern.ch/lepsusy/www/ sleptons_summer04/slep_final.html. 